\title{
Ensayo de una tipología de las cuencas mediterráneas del proyecto GUADALMED siguiendo las directrices de la directiva marco del agua.
}

\author{
Núria Bonada $^{1}$, Narcís Prat ${ }^{1}$, Antoni Munné ${ }^{1}$, Maria Rieradevall ${ }^{1}$, Javier Alba-Tercedor ${ }^{2}$, \\ Maruxa Álvarez ${ }^{3}$, Juan Avilés ${ }^{4}$, Jesús Casas ${ }^{5}$, Pablo Jáimez-Cuéllar ${ }^{2}$, Andrés Mellado $^{6}$, \\ Gabriel Moyà ${ }^{7}$, Isabel Pardo ${ }^{3}$, Santiago Robles ${ }^{4}$, Guillem Ramon ${ }^{7}$, M $^{\text {a }}$ Luisa Suárez ${ }^{6}$, \\ Manuel Toro ${ }^{4}, \mathrm{M}^{\mathrm{a}}$ Rosario Vidal-Abarca ${ }^{6}$, Soledad Vivas ${ }^{5}$ y Carmen Zamora-Muñoz ${ }^{2}$ \\ ${ }^{1}$ Departament d'Ecologia. Universitat de Barcelona. Diagonal, 645. 08028 Barcelona. \\ ${ }^{2}$ Departamento de Biología Animal y Ecología. Universidad de Granada. Campus Universitario de \\ Fuentenueva. 18071 Granada. \\ ${ }^{3}$ Área de Ecología. Universidad de Vigo. Campus Lagoas-Marcosende. 36200 Vigo. \\ ${ }^{4}$ CEDEX. División de Ecología de los Sistemas Acuáticos Continentales. Paseo Bajo Virgen del Puerto, 3. \\ 28005 Madrid. \\ ${ }^{5}$ Departamento de Biología Vegetal y Ecología. Universidad de Almería. Cañada de San Urbano, s/n. 04120 \\ Almería. \\ ${ }^{6}$ Departamento de Ecología e Hidrología. Universidad de Murcia. Campus de Espinardo. 30100 Murcia. \\ ${ }^{7}$ Departament de Biologia. Universitat de les Illes Balears. Crta. Valldemosa, km. 7.5. 07071 Palma de \\ Mallorca.
}

\section{RESUMEN}

Utilizando la propuesta de tipología de ríos de la Directiva Marco del Agua en su anexo 2 se realiza un ensayo tipológico de los puntos de muestreo del proyecto GUADALMED. Según el sistema A, se presentan 16 ecotipos, lo que se juzga poco adecuado para la gestión de los mismos y sin una base ecológica que lo justifique. De acuerdo con la selección previa hecha por los miembros del grupo GUADALMED para 5 de estos ecotipos no habría estaciones de referencia. Con algunas variables medidas en los puntos de muestreo y otras de tipo geológico e hidromorfológico, se realiza un ensayo tipológico usando el sistema B. El método implica un análisis de la correlación entre variables, un cluster de las estaciones utilizando el método K-means y un análisis discriminante de cuáles son las variables que distinguen estos grupos. El estudio da como resultado la caracterización de 6 ecotipos que sin embargo, a juicio de los miembros del proyecto GUADALMED, no recogen la variedad de tipos de ríos mediterráneos. Valorando cualitativamente parámetros no introducidos (por falta de datos) en el análisis K-means, se propone una tipología provisional en 9 grupos. Un análisis de las comunidades de macroinvertebrados (a nivel taxonómico de familia) revela un grado importante de coherencia entre las familias asociadas a cada tipo y sus preferencias ecológicas conocidas.

Palabras clave: Tipología, ecotipo, ecoregión, Sistema A, Sistema B, Directiva Marco del Agua

\begin{abstract}
A preliminary analysis was conducted of the river types in the GUADALMED project using the river typology proposed in the Water Framework Directive (WFD) in its annex 2. Using System A, 16 different types were found, a number too high for water management purposes and such typology lacks a supportive ecological basis. For 5 of these 16 ecotypes, no reference stations exist. Reference stations were selected a priori in project GUADALMED for each of the 12 basins sampled. An ecotypological analysis was performed using System B of the WFD using several variables measured during the study, and other variables obtained from maps or data bases (e.g., geology). The method included the analysis of the calibration between variables, the clustering of stations by the K-means method and a discriminant analysis. The latter revealed which factors were distinctive of each group. Six ecotypes were established for the GUADALMED study sites. These, however, do not include all the mediterranean-type streams of the region. This is mainly due to the absence in the data base of some important features of streams in the data base such as temporality or the dependence of flow on the presence of karstic aquifers, attributes
\end{abstract}


which could not be measured. A preliminary typology of 9 ecotypes was finally proposed. An IndVal test applied to these 9 ecotypes showed that there are indicator families of each ecotype.

Keywords: Tipology, ecotype, ecoregion, System A, System B, Water Frame Directive

\section{GLOSARIO}

Ecoregión: basada en la clasificación de la Directiva Marco del Agua, según la cual los puntos muestreados caerían dentro de la ecoregión ibero-macaronésica.

Ecotipo: clase dentro de una ecoregión.

\section{INTRODUCCION}

El establecimiento de un método para la evaluación de la calidad ecológica para los ecosistemas de aguas continentales incluye la medida de un conjunto de atributos entre los que se destacan: la obtención de los datos físico-químicos y biológicos, la clasificación de las estaciones, la selección de las estaciones de referencia y los tests para la validación del método mediante comparación de nuevas localidades. La clasificación de las estaciones (conocida como tipología) es un paso importante y previo en todos los sistemas de evaluación (Resh et al., 1995; Reynoldson et al., 1997) y ha sido utilizada por los científicos y gestores durante años para conseguir una organización de la información del sistema (Naiman et al., 1992). El desarrollo de métodos para las clasificaciones ha estado impulsado por una percepción generalizada de las similitudes y discontinuidades en las comunidades existentes entre tramos fluviales (Gerritsen et al., 2000).

En 1961 Illies desarrolló una metodología basada en parámetros físiográficos que se ha usado ampliamente en distintos países (Illies \& Botosaneanu, 1963). A partir de este trabajo los términos rhithron y potamon fueron utilizados de forma habitual en ecología de ríos durante un tiempo, aunque su uso actual es mínimo. Margalef (1983) realiza una buena síntesis y crítica de estos sistemas de clasificación iniciales. La posible variación de los tipos de ríos en función de la escala temporal y espacial utilizada ha sido incorporada más recientemente
(Frissell et al., 1986), y actualmente existen varias aproximaciones para la clasificación de los ríos, que se resumen en el uso de parámetros físicos, biológicos o ambos a la vez. En primer lugar están aquellas basadas en el uso de las ecoregiones preestablecidas (Omernik, 1995; Bailey, 1996) y también las que usan una clasificación jerárquica relacionando distintas escalas espaciales (Warren, 1979; Frissell et al., 1986). Otras aproximaciones utilizan a los organismos y están basadas en una clasificación de los sistemas fluviales usando la presencia y la abundancia de las comunidades de organismos (Hawkins \& Norris, 2000) e incluyendo parámetros físicos en el caso de los métodos conjuntos (Naiman et al., 1992). En Gerritsen et al. (2000) estas aproximaciones se resumen en dos tipos: las a priori, basadas en un conocimiento previo de las clases (ecoregiones, cuencas,...) o las a posteriori, donde no existe tal conocimiento ya que se parte de la comunidad de invertebrados para la clasificación (Wrigth et al., 1984; Moss et al., 1987). Además, los autores incluyen una tercera aproximación basada en testar las clases establecidas con los parámetros físicos con las comunidades biológicas. La predicción de las comunidades que se esperan en los puntos de muestreo y su comparación con los presentes en el momento de tomar las muestras es un método que se está desarrollando ampliamente en los últimos años (RIVPACS (Wrigth et al., 1993), BEAST (Reynoldson et al., 1995) o AusRivAS (Simpson et al., 1997), aunque su mayor limitación es que se requiere un elevado número de 
estaciones de referencia para la clasificación (Resh et al., 1995; Reynoldson et al., 1997).

El método a priori más utilizado actualmente para la clasificación de ríos es el uso de las ecoregiones (Omernik, 1995; Bailey, 1996), provincias biogeográficas (Hocutt \& Wiley, 1986) o una combinación de ambas (Abell et al., 2000). Aunque las ecoregiones están diseñadas con parámetros relativos a sistemas terrestres (vegetación, suelos, clima...) y se refieren a áreas grandes y limitadas, la relación de éstos con los acuáticos puede ser relevante, pues es necesario testar la concordancia de aquellas con las comunidades biológicas (Hynes, 1975). Para ello, varios son los estudios donde se comparan las ecoregiones con las distribuciones de peces, macroinvertebrados y algas (Pan et al., 2000). La mayoría de estos estudios muestran una poca correlación (Hawkins et al., 2000), hecho que podría ser atribuible a una gran variación dentro de la ecoregión (Resh et al., 1995; Hawkins \& Vinson, 2000; McCormick et al., 2000) y a la evidencia generalizada de que las comunidades pueden estar más controladas por factores locales que regionales (Hawkins \& Norris, 2000; Naiman et al., 2000; Sandin \& Johnson, 2000). Es por ello que algunos autores (Gerritsen et al., 2000; Hawkins et al., 2000) sugieren el establecimiento de subclases más pequeñas dentro de cada ecoregión delimitadas por factores locales, que incluyan toda la variabilidad de la ecoregión. El establecimiento de estas subclases, llamadas también ecotipos, implicaría la necesidad de establecer un mayor número de estaciones de referencia con el consiguiente aumento del coste económico si se usan en la gestión y la conservación de los sistema acuáticos. En cualquier caso, los criterios utilizados en la clasificación deben ser aquellos no influenciados o mínimamente por la actividad humanas, y que mejor ayuden a discriminar las clases (Munné \& Prat, 1999; 2000; Gerritsen et al., 2000).

La clasificación de los ecosistemas acuáticos es una parte importante y un paso previo en los estudios de seguimiento y evaluación del estado ecológico encaminados a la gestión de los ecosistemas que precisen del establecimiento de condiciones de referencia (Barbour et al., 1996; Hawkins et al., 2000; Van Sickle \& Hughes, 2000). Ello es debido a que las propiedades biológicas que se esperarían en los ríos sin la presencia de la alteración humana varían en función del tipo de río (Hawkins \& Norris, 2000; Oswood et al., 2000). Además, la clasificación es de gran utilidad en los Protocolos de Evaluación Rápida de la Calidad Ecológica (aquí referidos como PRECE, y más conocidos por sus siglas en inglés, RBP) (Resh et al., 1995; Norris, 1995) ya que así se obtiene una reducción de la variabilidad natural de los datos biológicos por clases, con lo que la determinación del estado ecológico puede ser más precisa y fiable (Gerritsen et al., 2000). Una vez tipificadas las estaciones, y establecidas las condiciones de referencia para cada ecotipo, se podrían detectar la respuestas de las comunidades a las perturbaciones en una estación, mediante comparación con los puntos de referencia de ese ecotipo (Feminella, 2000). Esta clasificación de las localidades permitiría además, una reducción del error debido a la extrapolación, tal y como se ha sugerido para las ecoregiones (Warry \& Hanau, 1993).

Debido a que las comunidades de macroinvertebrados y otros organismos pueden variar de manera natural en función de las características geomorfológicas del punto a estudiar, la Directiva Marco del Agua, en su Anexo 2, incide en que para una determinación precisa del estado ecológico se debe realizar una tipificación de las estaciones de muestreo utilizando parámetros geomorfológicos (D.O.C.E., 2000). Con esta tipificación, se obtendrían los ecotipos (en el sentido de Gerritsen et al., 2000; Hawkins et al., 2000) dentro de las distintas regiones limnológicas identificadas en la DMA y que son las propuestas por Illies (1978) en su Limnofauna Europea. Estos ecotipos deberían ser homogéneos en cuanto a características ambientales y biológicas. $\mathrm{La}$ DMA propone 2 métodos para la clasificación de los puntos de muestreos, basados en parámetros físicos y geomorfológicos. Uno, el Sistema A, se basa en tres atributos: la altitud, el tamaño de la cuenca y la geología, mientras que el Sistema B 
permite la inclusión de más atributos, algunos de ellos relacionados con las características propias del río y la cuenca. Una vez establecida esta clasificación de todas las estaciones, se establecerán condiciones de referencia así como comunidades tipo para cada grupo.

En España existe una gran tradición en los estudios de tipología de ecosistemas acuáticos

Tabla 1. Criterios especificados en la DMA para la tipología de ríos mediante el Sistema A y B. Criteria in the Water Framework Directive (WFD) for the establishment of a river typology according to Systems $A$ and $B$.

\begin{tabular}{|c|c|}
\hline & Descriptores SISTEMA A \\
\hline \multirow[t]{2}{*}{ Factores obligatorios } & $\begin{array}{l}\text { Altitud } \\
\qquad \begin{array}{l}\text { alto: }>800 \mathrm{~m} \\
\text { altura media: } 200-800 \mathrm{~m} \\
\text { tierras bajas: }<200 \mathrm{~m} \\
\text { Tamaño cuenca } \\
\text { pequeña: } 10-100 \mathrm{~km}^{2} \\
\text { mediana: }>100-1000 \mathrm{~km}^{2} \\
\text { grande: }>1000-10000 \mathrm{~km}^{2} \\
\text { muy grande: }>10000 \mathrm{~km}^{2} \\
\text { Geología } \\
\text { calcáreo } \\
\text { silíceo } \\
\text { orgánico }\end{array}\end{array}$ \\
\hline & Descriptores SISTEMA B \\
\hline Factores obligatorios & $\begin{array}{l}\text { altitud } \\
\text { latitud } \\
\text { longitud } \\
\text { geología } \\
\text { tamaño }\end{array}$ \\
\hline Factores optativos & $\begin{array}{l}\text { distancia desde el nacimiento del río } \\
\text { energía de flujo } \\
\text { anchura media del agua } \\
\text { profundidad media del agua } \\
\text { pendiente media del agua } \\
\text { forma y configuración del cauce } \\
\text { categoría de caudal } \\
\text { forma del valle } \\
\text { transporte de sólidos } \\
\text { capacidad de neutralización de ácidos } \\
\text { composición media del sustrato } \\
\text { cloruros } \\
\text { oscilación de la temperatura del aire } \\
\text { temperatura media del aire } \\
\text { precipitaciones }\end{array}$ \\
\hline
\end{tabular}

que está sintetizada en Margalef (1983), aunque en ríos no existen tantos estudios como en otros ecosistemas. Una propuesta de sectorización ecológica para las cuencas fluviales fue elaborada por Vidal-Abarca et al. (1990) para la cuenca del Segura, utilizando parámetros ambientales relativos al clima, morfometría fluvial, hidrología; sustratos geológicos y usos de suelo. Pero el primer ensayo de tipología en el sentido de la DMA, usando el Sistema B, se realizó en 1999 por Munné \& Prat. Nuestro trabajo se basa en gran medida en éste último. Por otra parte, Marchamalo \& García de Jalón (2000) hicieron un ensayo del Sistema A de la DMA para la Península Ibérica, incluyendo más categorías geológicas (materiales detríticos y inconsistentes) que lo que indica la DMA, obteniendo un total de 35 ecotipos.

Las estaciones muestreadas en el proyecto GUADALMED están englobadas en una única región biogeográfica, la ibero-macaronésica, establecida en Illies (1978), y por tanto el establecimiento de ecotipos se va a realizar dentro de la misma. Así pues, en este trabajo se realiza un primer intento de tipificación de las localidades muestreadas con el Sistema A y B, conocer cómo se distribuyen las estaciones de referencia, determinadas a priori por los expertos, dentro de cada tipo y determinar cuál es la comunidad tipo de cada grupo de estaciones. Se trata de un estudio previo que se pretende ampliar en la segunda fase del Proyecto incluyendo más estaciones de referencia para permitir el desarrollo de un método predictivo.

\section{MATERIAL Y MÉTODOS}

Los factores que deben ser utilizados para la tipificación de los ríos, tal y como marca la DMA (Tabla 1), fueron calculados para las 156 estaciones de muestreo incluidas en el proyecto GUADALMED (la descripción detallada de las estaciones se puede encontrar en Toro et al., este volumen) para cada uno de los dos sistemas que propone la DMA, el A y el B. Para el Sistema A, la altitud se obtuvo mediante los 
Tabla 2. Criterios seleccionados para la tipología de los ríos Mediterráneos muestreados en el proyecto GUADALMED según el Sistema $\mathrm{B}$ de la DMA. Criteria from the System B in the WFD selected to establish a river typology for the Mediterranean rivers sampled in the GUADALMED project.

OBLIGATORIOS

Geología. \% de roca silícica, calcárea y margas/sedimentarias en cada subcuenca y cuenca del punto de muestreo.

Tamaño. Área de la cuenca drenada.

Altitud. Valores de la base de datos obtenidos de un mapa 1:50000.

Latitud y Longitud. Se calcularon con las UTM.

\section{OPTATIVOS}

Distancia del nacimiento al río. Órdenes de los ríos a escala 1:250.000.

Anchura media. Rangos: 1m/1-10m / 10-100m

Forma del cauce. Valor del QBR del reverso de la hoja de campo.

Caudal. Media de los caudales medidos. Rangos: $<1$ 1/s / 1-10 1/s / 100-10001/s / >1000 1/s.

Composición del sustrato. Puntuación de cada tipo de sustrato de la primera versión del IH: 1 si es $>10 \%$ y 0 si es menor o igual.

mapas topográficos de cada cuenca; la geología se calculó estimando el \% aproximado de rocas calcáreas, silíceas y sustrato orgánico, utilizando mapas geológicos a escala 1:50.000 y el tamaño de la cuenca drenada se calculó mediante estimación de su superficie utilizando el método de comparación de pesadas. Para la altitud y el tamaño de la cuenca y para cada estación, se aplicaron los rangos propuestos en la DMA. Las estaciones se subdividieron en dos grupos, las de referencia seleccionadas a priori por los expertos y las que no se seleccionaron como de referencia.

A los factores usados en el Sistema A que son obligatorios en el B (Tabla 1) se les añadió el \% de margas y roca sedimentaria en la geología, debido a su importancia en algunas cuencas del sureste peninsular (Hurtado et al., 2001). Los demás factores obligatorios del Sistema B, la latitud y la longitud, fueron extrapolados utilizando las UTM de cada punto de muestreo. Como factores optativos del Sistema B se escogieron los mostrados en la Tabla 2 que eran los que se pudieron calcular con los datos que poseíamos. La distancia del nacimiento al río se calculó mediante el orden del río a escala 1:250000; para la anchura y el caudal medios se establecieron unos rangos de acuerdo con los valores medidos en las campañas de muestreo (Tabla 2); y la forma del cauce fue calculada mediante el valor obtenido en el reverso de la hoja del QBR que indica la pendiente de ambos márgenes del punto estudiado (Munné et al., 1998, 2003) (ver artículo de Suárez et al., este volumen). Otros parámetros optativos propuestos en la DMA (Tabla 1) no pudieron ser utilizados.

Para realizar una tipificación mediante el Sistema B, primero se midieron las correlaciones entre las variables utilizadas, para descartar o simplificar aquellas que estuvieran más correlacionadas. La medida de correlación utilizada fue la rho de Spearman debido a que utilizamos parámetros cuantitativos y cualitativos, conjuntamente. A continuación, se clasificaron las localidades mediante estos parámetros geomorfológicos y físicos (previamente estandarizados), mediante una agrupación clúster. Debido a que no se conoce el número óptimo de ecotipos, se realizó una agrupación no-jerárquica con el método K-means. Este método, establece agrupaciones de localidades mediante las variables entradas, en función del número de clases o grupos que se especifican y maximizando las diferencias entre grupos. Así, realizamos este análisis con $6,7,8$ y 9 grupos. Una vez clasificadas las estaciones en estos grupos, se aplicó un análisis discriminante paso a paso con el método Lambda de Wilks. Este análisis permite conocer las variables de más a menos significativas para discriminar las diferencias entre los 
grupos establecidos por el K-means. El estadístico Lambda de Wilks toma valores entre 0 y 1 , donde 0 indica que los grupos que se están comparando son distintos mientras que 1 indica lo contrario. Así, el análisis localiza aquellas variables que paso a paso, y junto con las variables que hayan sido ya seleccionadas, minimicen la Lambda de Wilks global. No obstante, que una variable sea seleccionada, es decir que tenga un valor de Lambda de Wilks mínimo, no significa que sea considerada como discriminante, ya que hay que compararla con el estadístico $\mathrm{F}$ que indica la variación de Lambda de Wilks que se produciría si se seleccionara. Así, un valor de $\mathrm{F}$ grande indica que la variable tiene mucho peso en el Lambda de Wilks y por tanto es correcto el seleccionarla. Para establecer un criterio en esta selección de variables discriminantes, se fija el valor crítico de F de entrada de 3.84 , lo que indica que las variables que tomen un valor de $\mathrm{F}>3.84$ serán discriminantes, y las que presenten un $\mathrm{F}<3.84$ no a pesar de tener un Lambda de Wilks pequeño.

Después de conocer las variables más significativas para diferenciar los grupos, se seleccionó la agrupación que mostraba una mejor clasificación de las estaciones, desde el punto de vista ecológico, y continuidad espacial. Con ello, se obtuvo una tipología donde cada estación se relacionaba con uno de los ecotipos. Para cada ecotipo, se analizaron las características de las estaciones de referencia previamente seleccionadas. Los resultados se muestran en Bonada et al (este volumen). Los análisis estadísticos se realizaron utilizando el SPSS vs.9.0.1. La tipología resultante fue estudiada por parte del grupo GUADALMED, y de acuerdo con la experiencia de los diferentes equipos de trabajo se propusieron modificaciones que no podían ser contempladas por el análisis por falta de datos. Se empleó así otra aproximación sugerida en la DMA (panel de expertos) cuando no existan suficientes datos para realizar una tipología definitiva.

Una vez obtenida la tipología con el Sistema B reformada con la opinión de los expertos, para cada ecotipo se analizó la comunidad tipo utilizando los datos correspondientes a todas las cam- pañas del proyecto GUADALMED. Ello se realizó aplicando el programa estadístico IndVal (Dufrêne \& Legendre, 1997) que trata de buscar "especies indicadoras" (familias en nuestro caso) de cada grupo de estaciones preestablecido.

\section{RESULTADOS}

\section{Tipología mediante el Sistema A}

De los 32 grupos posibles que pueden resultar de una tipificación mediante el Sistema A, nuestros datos indicaron la existencia de 16 ecotipos, ya que sólo se detectó la presencia de dos tipos de síntesis (la categoría de síntesis "orgánicos" no se presenta en nuestros ríos). En el Anexo 1 se muestran las localidades distribuidas por los ecotipos resultantes. Tal y como se observa algunos de ellos están formados por tan sólo 1 estación ("Alto, Mediano, Silíceo" y "Altura media, Muy grande, Calcáreo"), hecho que puede dificultar el establecimiento de condiciones de referencia dentro de ese ecotipo. Algunos ecotipos son imposibles en las cuencas mediterráneas, sobretodo los referidos a la geología orgánica (presencia de turba) o a aquellas situaciones que difícilmente podrían existir dadas las características de la zona, como "Altos, Muy grandes, silíceos o calcáreos".

En el Anexo 1, también se puede observar la distribución de las localidades en los distintos ecotipos en función de si fueron seleccionadas de referencia o no por los expertos. Se observa que solamente 11 ecotipos de los 16 establecidos, poseerían estaciones de referencia seleccionadas según los expertos. Los restantes, corresponden o bien a ecotipos que con pocas estaciones totales (como el ecotipo, "Alto, Medio, Silíceo") y / o a ríos medianos o grandes, donde fue difícil establecer estaciones de referencia debido a la elevada alteración de estas zonas.

\section{Tipología mediante el Sistema B}

Para evitar que localidades altamente correlacionadas expresaran cierta redundancia y por lo tanto, tuvieran demasiado peso al discriminar los ecotipos, se calcularon las correlaciones de 
Spearman entre todos los factores (Tabla 3). El resultado fue que, a pesar de que salieron muchas correlaciones significativas, tan sólo algunas de las relacionadas con la geología presentaban coeficientes de correlación elevados $(|r>0.8|)$, especialmente al comparar la subcuenca con la cuenca. Debido a ello, se eliminaron las variables geológicas de subcuenca, debido a que, de manera general, se entiende que es toda la cuenca la que afecta a un punto de muestreo y no tan sólo la subcuenca. Además, se observa que también existe una elevada correlación negativa entre la cuenca silícea y la calcárea. Todo ello fue debido al uso de porcentajes entre las distintas categorías del factor geológico (silíceo vs. calcáreo vs. sedimentario).

Debido a que geología de cuenca estaba representada por 3 variables, y para evitar que hubiera un mayor peso de la geología respecto a las demás variables, se unificó el valor de la variable geología mediante un Análisis de Componentes Principales (Ter Braak, 1988) siguiendo la metodología de Munné \& Prat (1999, 2000). El resultado de este análisis mostró que las 3 categorías geológicas de cuenca se podían simplificar en dos, correspondientes a los ejes $\mathrm{X}$ e $\mathrm{Y}$ del análisis (Fig. 1). El primer eje (que explica un $68.8 \%$ de la variabilidad de los datos) indicaría un eje silíceo-calcáreo (simplificando así estas dos variables altamente correlacionas en una sola), mientras que el segundo (31.2\% de la variabilidad) sería sedimentariono-sedimentario. Los valores de las proyecciones de las estaciones sobre el eje $\mathrm{X}$ e $\mathrm{Y}$ fueron estandarizados y utilizados como nuevas variables: X_PCA relacionado con la geología más o menos silícea de la cuenca y el Y_PCA indicando su carácter más o menos sedimentario.

Utilizando los valores del análisis del PCA para las variables geológicas y todas las demás variables, se aplicó el método K-means para la obtención de grupos de estaciones. Los grupos se preestablecieron en $6,7,8$ y 9 clases. Posteriormente a los análisis discriminantes, el grupo 6 pareció ser el ecológicamente y espacialmente más razonable en las estaciones muestreadas, ya que a partir de 6 grupos los puntos de cada ecoti-

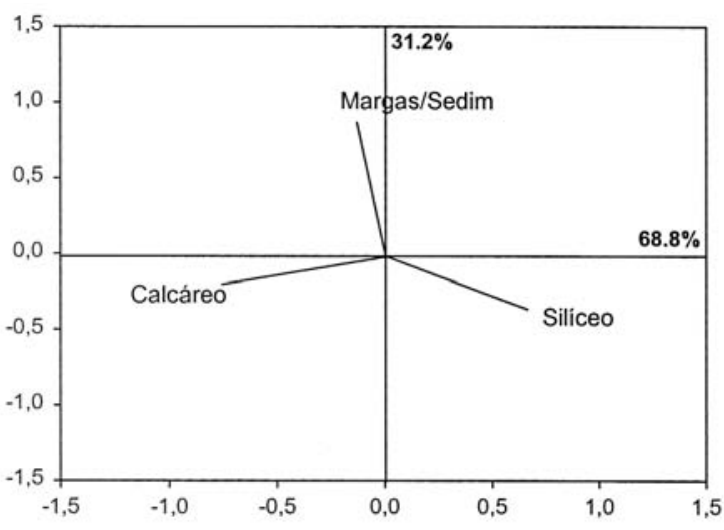

Figura 1. PCA con la geología de la cuenca de todas las estaciones, expresada en \%. El primer eje proporciona un $68.8 \%$ de la variabilidad total de los datos, mientras que el segundo un $31.2 \%$. PCA of basin geology (in \%) for all sampling stations. The first axis of the PCA explains $68.8 \%$ of total data variability, while the second axis can explain $31.2 \%$.

po tenían una distribución muy irregular o bien se discriminaba mucho para unos ecotipos y muy poco para otros (pocas estaciones por ecotipo).

Para conocer el significado ecológico de cada grupo, y para con ello caracterizar los ecotipos definitivos, se aplicó el análisis discriminante paso a paso (método Lambda de Wilks), mediante el que se seleccionaron las variables que mejor ayudaban a discriminar los distintos grupos. Así, según muestra la Tabla 4, las variables con valor de Lambda de Wilks pequeño y F $>3.84$ son: El ancho del cauce, los factores relacionados con la geología, el orden del río, la forma del cauce, el caudal, la temperatura en primavera y la altitud. Por su parte, la latitud no aparece como significativa para diferenciar los tipos (tiene un Lambda de Wilks pequeño pero una $\mathrm{F}<3.84$ ), ya que las características ambientales de una estación, pueden repetirse en otras lejanas a ella; por ejemplo las características que hallamos en los ríos que fluyen a través del Montseny en el NE de España (cuenca del Besós) son parecidas a las que fluyen en las cabeceras de los ríos de Sierra Nevada en el SE (como el Adra o el Guadalfeo). El área de la cuenca y el sustrato parecen ser también menos relevantes para discriminar los tipos según este análisis. 
Tabla 4. Valores de Lambda de Wilks y F para cada variable y cada paso. En sombreado se muestran las variables seleccionadas y significativas en cada paso, que son las que discriminan mejor los 6 grupos resultantes del K-means. Wilks' Lambda and $F$ values for each variable and step. In bold are selected variables which are significant in each step. These variables are those better discriminating the 6 groups resulting from the K-means analysis.

\begin{tabular}{|c|c|c|c|c|c|c|c|}
\hline & $\begin{array}{l}\text { Variables } \\
\text { seleccionadas }\end{array}$ & $\begin{array}{l}\text { F que } \\
\text { introducir }\end{array}$ & $\begin{array}{l}\text { Lambda } \\
\text { deWilks }\end{array}$ & & $\begin{array}{l}\text { Variables } \\
\text { seleccionadas }\end{array}$ & $\begin{array}{l}\text { F que } \\
\text { introducir }\end{array}$ & $\begin{array}{l}\text { Lambda } \\
\text { deWilks }\end{array}$ \\
\hline \multirow[t]{11}{*}{ Paso 0} & ANCHO & 2521.9 & 0.012 & & ALTITUD & 12.16 & 0 \\
\hline & X-PCA & 261.9 & 0.103 & & CAUDAL & 9.6 & 0 \\
\hline & Y-PCA & 84.9 & 0.261 & & SUSTRATO & 2.4 & 0 \\
\hline & AREA & 35.8 & 0.456 & & LATITUD & 1.8 & 0 \\
\hline & ORDEN & 23.75 & 0.558 & & AREA & 1.4 & 0 \\
\hline & TEMPERATURA & 23.8 & 0.558 & & & & \\
\hline & FORMA & 17.9 & 0.626 & \multirow[t]{7}{*}{ Paso 4} & FORMA & 12.5 & 0 \\
\hline & LATITUD & 17.9 & 0.627 & & TEMPERATURA & 10.8 & 0 \\
\hline & CAUDAL & 16.3 & 0.648 & & CAUDAL & 8.2 & 0 \\
\hline & ALTITUD & 14.3 & 0.677 & & ALTITUD & 6.3 & 0 \\
\hline & SUSTRATO & 8.4 & 0.78 & & LATITUD & 3.3 & 0 \\
\hline \multirow[t]{10}{*}{ Paso 1} & X-PCA & 248.3 & 0.001 & & AREA & $\begin{array}{l}2.8 \\
0.7\end{array}$ & 0 \\
\hline & Y-PCA & 85.4 & 0.003 & & & & \\
\hline & TEMPERATURA & 24.9 & 0.006 & \multirow[t]{7}{*}{ Paso 5} & CAUDAL & 6.9 & 0 \\
\hline & LATITUD & 17.5 & 0.007 & & TEMPERATURA & 6.6 & 0 \\
\hline & FORMA & 18.6 & 0.007 & & ALTITUD & 5 & 0 \\
\hline & ALTITUD & 18.8 & 0.007 & & SUSTRATO & 2.8 & 0 \\
\hline & ORDEN & 23.5 & 0.007 & & LATITUD & 1.9 & 0 \\
\hline & CAUDAL & 14.5 & 0.008 & & AREA & 0.6 & 0 \\
\hline & SUSTRATO & 8.2 & 0.009 & & & & \\
\hline & AREA & 1.3 & 0.011 & \multirow[t]{4}{*}{ Paso 6} & TEMPERATURA & 7.8 & 0 \\
\hline \multirow[t]{9}{*}{ Paso 2} & Y-PCA & 85.4 & 0 & & $\begin{array}{l}\text { ALTITUD } \\
\text { SUSTRATO }\end{array}$ & $\begin{array}{l}4.6 \\
3.2\end{array}$ & $\begin{array}{l}0 \\
0\end{array}$ \\
\hline & TEMPERATURA & 23.5 & 0.001 & & LATITUD & 2.3 & 0 \\
\hline & ORDEN & 23.4 & 0.001 & & AREA & 0.9 & 0 \\
\hline & ALTITUD & 18.9 & 0.001 & & & & \\
\hline & FORMA & 15.6 & 0.001 & \multirow[t]{4}{*}{ Paso 7} & ALTITUD & 4.7 & 0 \\
\hline & CAUDAL & 12.9 & 0.001 & & SUSTRATO & 3.4 & 0 \\
\hline & SUSTRATO & 6.9 & 0.001 & & LATITUD & 2.3 & 0 \\
\hline & LATITUD & 2.7 & 0.001 & & AREA & 0.9 & 0 \\
\hline & AREA & 1.3 & 0.001 & \multirow{4}{*}{ Paso 8} & & & \\
\hline \multirow[t]{3}{*}{ Paso 3} & ORDEN & 22.6 & 0 & & LATITUD & $\begin{array}{l}3.0 \\
2.4\end{array}$ & 0 \\
\hline & FORMA & 17.3 & 0 & & AREA & 1.5 & 0 \\
\hline & TEMPERATURA & 13.5 & 0 & & & & \\
\hline
\end{tabular}

En la figura 2 se muestran unos diagramas de cajas para que, una vez conocidas las variables que mejor discriminan los 6 ecotipos, ayudar a establecer el significado de cada una de ellos. Así se observa que la variable "anchura media" discrimina muy bien el ecotipo 5 del resto, que a la vez también está asociado a un elevado caudal y a una cuenca muy poco silícea. En la figura $2 b$ y $2 \mathrm{c}$ observamos que la geología permite diferenciar bien los ecotipos 2,3 y 6 del resto, de manera que los dos primeros están formados por estaciones con cuenca silícea mientras que el 6 tienen una cuenca de tipo sedimentario. Mientras, en los demás ecotipos domina el sustrato calcáreo. Por su parte el factor indicativo de la distancia del nacimiento al río (orden) (Fig. 2d) es altamente variable en todos los ecotipos, pero el ecotipo 3 y especialmente el 5 poseen un orden mayor. La temperatura del agua (Fig. $2 \mathrm{~g}$ ) es importante para separar las estaciones del ecotipo 6 del resto, que además parece ser que posee una altitud muy baja al contrario de los ecotipos 

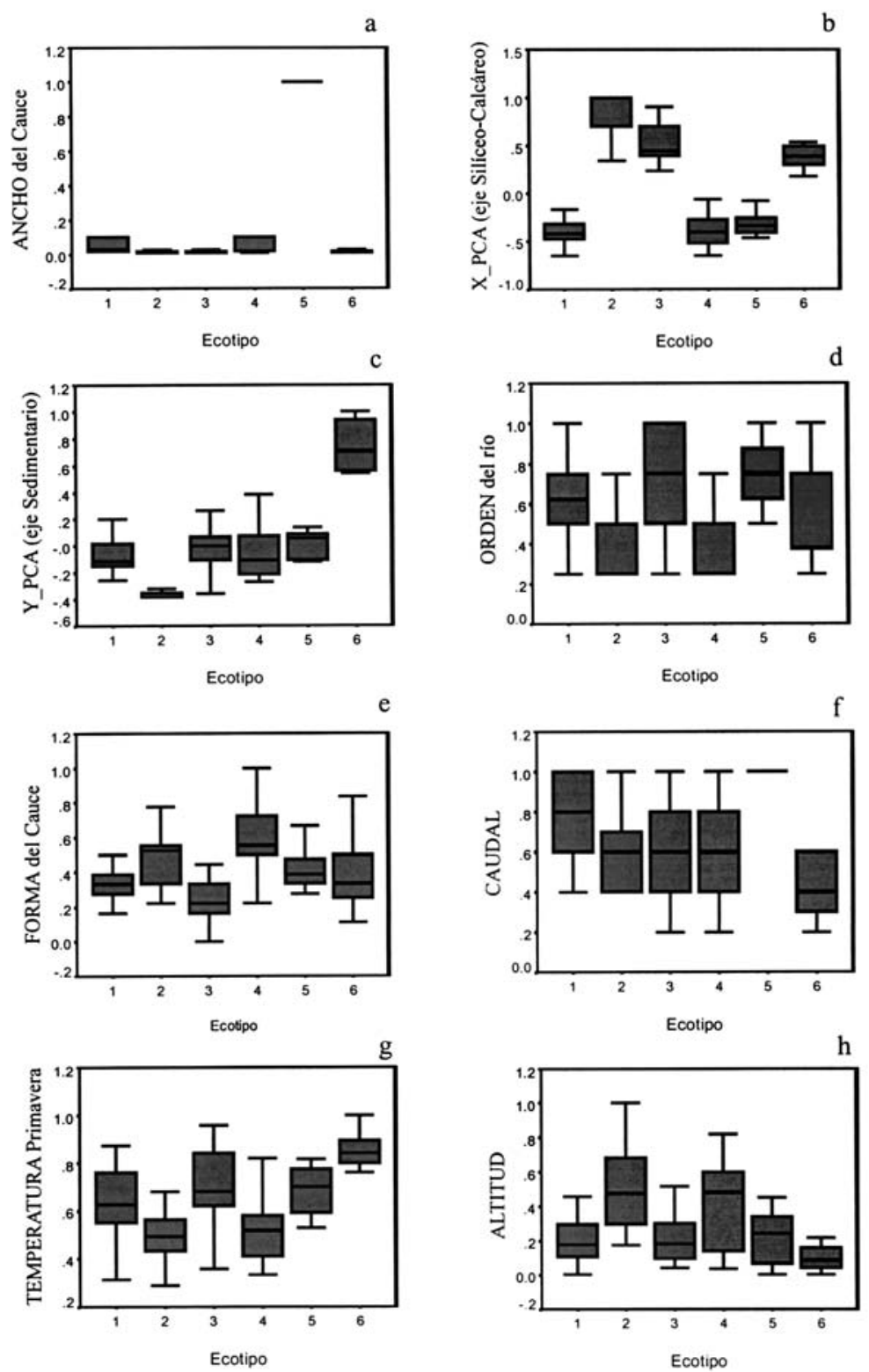

Figura 2. Diagrama de cajas para cada variable discriminante según los ecotipos hallados en el Sistema B. Box Plots for each discriminant variable according to the ecotypes in System $B$.

2 y 4. Por otra parte, el factor "forma" (Fig. 2e) presenta valores elevados en las cabeceras calcáreas y silíceas, tal y como era de esperar pues estas zonas poseen márgenes laterales con relativa pendiente, propios de ríos de montaña. Así pues, los 6 ecotipos tendrían el significado ecológico que se resume en la Tabla 5. Esta caracterización de cada ecotipo de manera simplificada está basada en las cuatro variables más significativas para discriminar los grupos (ancho, X_PCA, Y_PCA y orden del río).

En el Anexo 2 se muestra un listado de las estaciones distribuidas por ecotipos en función de los resultados del K-means. Además, se indican, para cada tipo, las estaciones seleccionadas como de referencia. Tal y como se observa, encontramos 
Tabla 5. Ecotipos resultantes para el Sistema B, clasificados según el tamaño del río, la geología dominante en la cuenca y la posición en el eje del río o afluente. La columna código, resume el significado de cada ecotipo e indica la nomenclatura utilizada para su representación en los anexos. Ecotypes obtained for System B, classified according to river size, basin dominant geology and position in the river or tributary. The code field summarizes the meaning of each ecotype and indicates the names used in annexes.

\begin{tabular}{lllll}
\hline & TAMAÑO del río & GEOLOGÍA cuenca & POSICIÓN & Código \\
\hline ECOTIPO 1 & Mediano & Calcáreo & Medio-Bajo & $\mathrm{Ca1} / \mathrm{MB}$ \\
ECOTIPO 2 & Pequeño & Silíceo & Cabecera & $\mathrm{Sil} / \mathrm{Cab}$ \\
ECOTIPO 3 & Mediano & Silíceo & Medio-Bajo & $\mathrm{Sil} / \mathrm{MB}$ \\
ECOTIPO 4 & Pequeño & Calcáreo & Cabecera & $\mathrm{Cal} / \mathrm{Cab}$ \\
ECOTIPO 5 & Grande & Calcáreo/Sedimentario & Bajo & $\mathrm{Cal} / \mathrm{GB}$ \\
ECOTIPO 6 & Ramblas & Sedimentario & & Ramblas \\
\hline
\end{tabular}

estaciones de referencia según los expertos en cada ecotipo, hecho que indicaría la posibilidad de establecer el buen estado ecológico o el potencial ecológico máximo, para cada ecotipo, y con ello fijar los rangos de calidad.

Utilizando las coordenadas UTM y los mapas de las cuencas muestreadas se construyó la figura 3 , donde se localizaron las estaciones separadas por ecotipos. Ello permite ver, de una manera clara, la distribución de los ecotipos a lo largo del mediterráneo. Así, observamos que los tramos medios-bajos de ríos grandes se hallan a lo largo del eje del Júcar y de sus afluentes más importantes. Las Ramblas están confinadas a las cuencas del Segura, Aguas y Almanzora, mayoritariamente, zonas altamente dominadas por margas y sustrato sedimentario. En cambio las cabeceras silíceas y los tramos medios-bajos de ríos medianos de cuencas silíceas son los que fluyen de Sierra Nevada y Montseny. En general, se observa que el ecotipo más ampliamente distribuido es el relacionado con una geología calcárea, típica del levante peninsular. Así, encontramos cuencas exclusivamente calcáreas (Llobregat, Millars, Turia, Pollença y Sóller) donde todas las localidades se agrupan en los 2 ecotipos calcáreos.

A pesar de que a nivel general los ecotipos obtenidos resultaron ser bastante coherentes con la opinión que tenían los expertos de cada uno de los puntos, algunas estaciones fueron mal clasificadas. Este fue el caso, por ejemplo, de todo el eje del Júcar que según el análisis pertenecía a el ecotipo "Ríos Grandes", con lo cual el río no tendría "tramos medios-bajos", o al Aguas cuyas estaciones fueron clasificadas en el ecotipo "Ramblas" debido a su elevada composición en materiales de tipo sedimentario y sin embargo algunas de sus partes tienen caudal permanente, lo cual no es propio de las ramblas. Además de esto, en algunas estaciones presentaban características (como por ejemplo la temporalidad, el estar situadas en la llanura aluvial o el ser alimentadas por fuentes en zonas carstificadas) que no quedaron recogidas en los diferentes ecotipos al no haber considerado estos factores, pero que podían tener implicaciones en la estructura y composición de las comunidades de macroinvertebrados. Los seis ecotipos establecidos por el análisis eran, a juicio de la opinión de los integrantes del proyecto GUADALMED, insuficientes para describir los "ecotipos" que parecían deducirse la información adyacente en los estudios de comunidades.

Después de intensas discusiones por parte del grupo y de la imposibilidad de realizar de nuevo el análisis estadístico (pues no se disponía de datos de temporalidad o de caudales relacionados con las fuentes) se propuso establecer una tipología mixta que a la vez reflejara los resultados del análisis con el cluster Kmeans y la percepción (experiencia) de los integrantes del grupo. Con ello, se utilizaba la misma metodología ("expert panelling") que se usa en algunos aspectos de la gestión de aguas cuando la información previa es insuficiente o insatisfactoria y que se prevé también en la DMA. Esta tipología deberá considerarse como una tipología previa a la definitiva que se reali- 


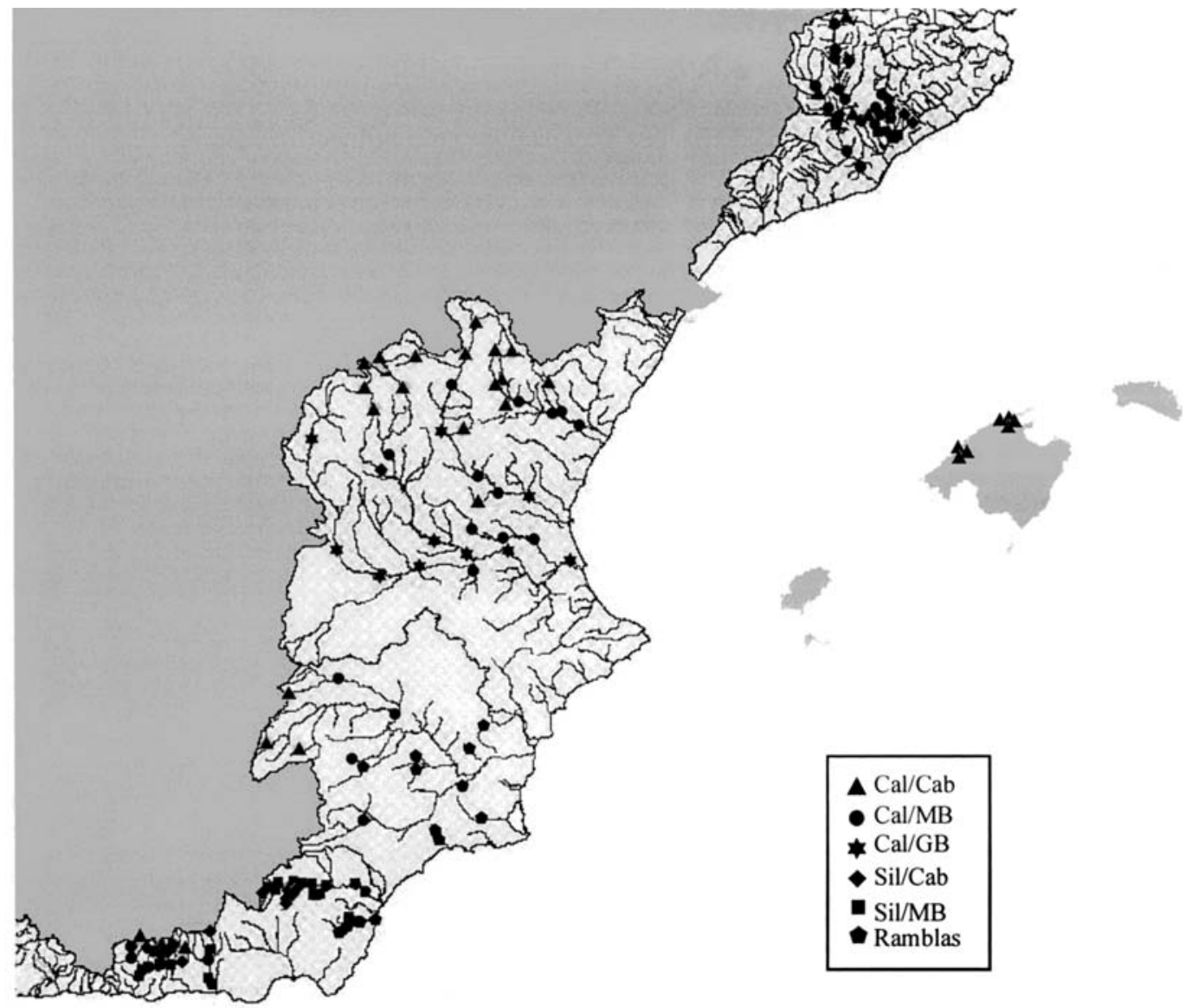

Figura 3. Localización en el espacio de los distintos ecotipos resultantes del Sistema B. Distribution in physical space of the obtained ecotypes according to System $B$.

zará en el marco de la segunda fase del proyecto GUADALMED.

Con este enfoque, se establecieron tres nuevos ecotipos, que si bien no estaban fundamentados con los resultados estadísticos, englobarían estaciones con características hidrológicas y biológicas peculiares y diferentes a las de los demás tipos. Así, se establecieron los ecotipos "Temporales" y "Karst", que pretendían diferenciar las estaciones que se secaban de forma habitual en algún momento del año (por lo menos 3 meses) y las que estaban alimentadas por fuentes en caudal continuo de zonas carstificadas ("karsts"). Además, se añadió el ecotipo "Llanura Aluvial", para englobar aquellas estaciones correspondientes a los tramos más bajos del río Júcar, de características geomorfológicas muy distintas a las de los tramos bajos de las demás cuencas y a las zonas medias y bajas del propio Júcar. A estos nuevos ecotipos establecidos por los componentes del proyecto actuando como expertos, se les asignaron las estaciones correspondientes, que se muestran en el Anexo 3.

Para cada uno de los nueve ecotipos delimitados se determinó la comunidad más característica mediante la aplicación del programa IndVal al 0.05 de error. Los resultados de este análisis se muestran en la Tabla 6 . Tal y como se observa, taxones muy comunes, como pueden ser los quironómidos, no aparecen significativos de ningún ecotipo, puesto que se pueden hallar en cualquiera. No obstante, otros, parecen ser exclusivos de algún grupo de estaciones. Así lo son para las cabeceras silíceas, los blefarocéridos, braquicéntridos, heptagénidos y la mayoría de los plecópteros y tricópteros típicos de aguas frías, entre otros, mientras que para el 
Tabla 6. Resultados del IndVal para todas las campañas de muestreo. Se representan las familias más significativamente presentes en cada ecotipo, al $\mathrm{p}=0.05$, juntamente con su valor indicador (IV) ordenado. IndVal results for all sampling occasions. Families with most significant presence in each ecotype at $p=0.05$ and their indicator value (IV) are presented.

\begin{tabular}{|c|c|c|c|c|c|c|c|}
\hline Calc/Cab & IV & $\mathrm{Cal} / \mathrm{MB}$ & IV & Cal/GB & IV & Sil/Cab & IV \\
\hline Elmidae & 58.8 & Physidae & 61.7 & Heptageniidae & 71 & Limoniidae & 70.5 \\
\hline Limnephilidae & 58.2 & Hydropsychidae & 56.7 & Atyidae & 69.7 & Rhyacophilidae & 67.1 \\
\hline Gerridae & 56 & Baetidae & 54.7 & Empididae & 62.5 & Ephemerellidae & 64.7 \\
\hline Nemouridae & 50.1 & Ostracoda & 54.5 & Potamanthidae & 60.5 & Nemouridae & 64.4 \\
\hline Leuctridae & 48.6 & Caenidae & 54.4 & Hydracarina & 60.2 & Perlidae & 60.8 \\
\hline Stratiomyidae & 48 & Hydracarina & 48.4 & Caenidae & 60.1 & Heptageniidae & 57.1 \\
\hline Perlidae & 47.6 & Hydroptilidae & 46.4 & Hydropsychidae & 59.7 & Limnephilidae & 56.4 \\
\hline Heptageniidae & 45.6 & Gerridae & 44 & Gammaridae & 55.1 & Dytiscidae & 55.6 \\
\hline Ephemerellidae & 45.5 & Corixidae & 43.9 & Dugesiidae & 50.3 & Simuliidae & 55.3 \\
\hline Helodidae & 43.4 & Hydrobiidae & 41.1 & Polymitarcidae & 48 & Tabanidae & 54.9 \\
\hline Gomphidae & 42.3 & Ancylidae & 40.9 & Sphaeriidae & 42.8 & Elmidae & 51.6 \\
\hline Psychodidae & 42.3 & Erpobdellidae & 39.9 & Calopterygidae & 40.7 & Tipulidae & 50.9 \\
\hline Hydrobiidae & 42.2 & Hydrometridae & 35.5 & Oligoneuriidae & 35.5 & Dixidae & 50.2 \\
\hline Haliplidae & 42 & Dugesiidae & 30.4 & Neritidae & 34.2 & Sericostomatidae & 49.3 \\
\hline Empididae & 41.2 & Gomphidae & 28.3 & Prosopistomatidae & 25 & Brachycentridae & 48.8 \\
\hline Sphaeriidae & 40.6 & Athericidae & 23.4 & & & Empididae & 48.7 \\
\hline Ephemeridae & 39.2 & Platycnemididae & 20.2 & & & Perlodidae & 46.8 \\
\hline Sericostomatidae & 38.6 & Astacidae & 13.7 & & & Glossiphoniidae & 45.6 \\
\hline Calopterygidae & 37.2 & & & & & Leuctridae & 44.7 \\
\hline Leptophlebiidae & 35.2 & & & & & Cordulegasteridae & 42.2 \\
\hline Cordulegasteridae & 34.5 & & & & & Polycentropodidae & 41.4 \\
\hline Perlodidae & 34.4 & & & & & Dryopidae & 37.6 \\
\hline Gyrinidae & 33.9 & & & & & Philopotamidae & 35.7 \\
\hline Aeshnidae & 31.9 & & & & & Planariidae & 35.4 \\
\hline Sialidae & 30.3 & & & & & Capniidae & 35.1 \\
\hline Lepidostomatidae & 27.9 & & & & & Blephariceridae & 31.2 \\
\hline Bythinellidae & 27.4 & & & & & Chloroperlidae & 26.7 \\
\hline Athericidae & 25 & & & & & Lepidostomatidae & 25.6 \\
\hline Taeniopterygidae & 19.2 & & & & & Helophoridae & 21.4 \\
\hline Lestidae & 16 & & & & & Osmylidae & 12.5 \\
\hline Valvatidae & 13.1 & & & & & & \\
\hline Capniidae & 12.7 & & & & & & \\
\hline Siphlonuridae & 10.5 & & & & & & \\
\hline Viviparidae & 10.5 & & & & & & \\
\hline Sil/MB & IV & Karst & IV & Ramblas & IV & Temporal & IV \\
\hline Lymnaeidae & 47.9 & Polycentropodidae & 72.5 & Hydrophilidae & 76.5 & Ostracoda & 58.6 \\
\hline Culicidae & 33.2 & Hydraenidae & 66.1 & Coenagrionidae & 68.7 & Dytiscidae & 52 \\
\hline \multirow[t]{21}{*}{ Oligoneuriidae } & 14 & Elmidae & 63.2 & Corixidae & 67.5 & Stratiomyidae & 51 \\
\hline & & Ceratopogonidae & 63.1 & Naucoridae & 63.3 & Hydraenidae & 46.5 \\
\hline & & Caenidae & 62.7 & Notonectidae & 62.6 & Limnephilidae & 45.3 \\
\hline & & Stratiomyidae & 60.5 & Libellulidae & 62 & Anthomyidae & 37.3 \\
\hline & & Tabanidae & 60.5 & Aeshnidae & 60.8 & Dolichopodidae & 27.2 \\
\hline & & Hydroptilidae & 58.6 & Dytiscidae & 58.5 & Asellidae & 22.4 \\
\hline & & Ostracoda & 57.7 & Hydraenidae & 56.7 & Hygrobiidae & 17.2 \\
\hline & & Dixidae & 55 & Ceratopogonidae & 55.2 & & \\
\hline & & Rhyacophilidae & 53.1 & Pleidae & 53 & & \\
\hline & & Leuctridae & 52.7 & Gammaridae & 50.4 & Llan-Aluvial & IV \\
\hline & & Libellulidae & 52.6 & Hydrobiidae & 48.8 & & \\
\hline & & Glossosomatidae & 52.4 & Dryopidae & 47.3 & & \\
\hline & & Planorbidae & 51.9 & Thiaridae & 46.9 & & \\
\hline & & Nepidae & 50.8 & Cambaridae & 43.6 & & \\
\hline & & Haliplidae & 49.6 & Stratiomyidae & 43.2 & & \\
\hline & & Notonectidae & 47.9 & Dolichopodidae & 41.7 & & \\
\hline & & Anthomyidae & 45.4 & Ephydridae & 40.4 & & \\
\hline & & Psychomyidae & 44.3 & Nepidae & 39.4 & & \\
\hline & & Leptoceridae & 36.1 & Culicidae & 37.2 & & \\
\hline & & Dolichopodidae & 35.4 & Noteridae & 27.2 & & \\
\hline & & Hydrochidae & 24.1 & Neritidae & 18.2 & & \\
\hline
\end{tabular}


ecotipo de cabeceras calcáreas, aparecen como taxones de manera más significativa algunos odonatos, coleópteros, algunos tricópteros y algún plecóptero como los leúctridos, pérlidos y perlódidos, entre otros.

Las comunidades propias de los tramos mediosbajo de ríos medianos silíceos están formadas por limnaeidos y oligoneuriellidos, mientras que cuando la cuenca es calcárea, aparecen los astácidos, caénidos, erpobdéllidos, hidropsíquidos, así como algunos heterópteros y moluscos.

En los tramos medios-bajos de ríos grandes calcáreos, organismos propios de estos hábitats aparecen como significativos, como podrían ser los atíidos, nerítidos, oligoneuriéllidos, potamántidos o los prosopistomátidos. En cambio en los sistemas tipo ramblas abundan de manera significativa los odonatos, coleópteros, heterópteros, tiáridos, propios de hábitats leníticos.

En los ríos de carácter temporal son abundantes algunas familias de coleópteros como los hydraénidos co los hygróbidos, juntamente con los limnefílidos del género Mesophylax sp., con adaptaciones de ciclo de vida propias de estos ambientes (Bouvet, 1974). Por otro lado, en la presencia de zonas karstificadas, que poseen pozas durante alguna época del año, los coleópteros y heterópteros también son significativos, juntamente con algunos tricópteros, tales como los psicómidos.

\section{DISCUSIÓN}

Muchas son las metodologías que tradicionalmente se han utilizado en la clasificación de los ríos, basándose en parámetros físicos y/o incluyendo otros más biológicos (Naiman et al., 1992). Para estos últimos casos, se han utilizado los peces (McCormick et al., 2000; Oswood et al., 2000), los macroinvertebrados (Wrigth et al., 1984; Moss et al., 1987; Rabeni \& Doisy, 2000), las algas (Pan et al., 2000) o la vegetación de ribera (por ejemplo Baker, 1989). Además, en Estados Unidos (Hawkins et al., 2000) y también en algunos países europeos (Sandin \& Johnson, 2000), el uso de la ecore- giones basadas en parámetros más terrestres han sido utilizadas, para conocer su utilidad para la clasificación de los sistemas fluviales (resumen en Hawkins et al., 2000). No obstante, la mayoría de estos estudios, sugieren que para mejorar la clasificación, distintos factores locales de cada tramo deberían ser incluidos (Resh et al., 1995; Hawkins \& Norris, 2000; Sandin \& Johnson, 2000), ya que a pesar de la relación existente entre los ecosistemas terrestres y acuáticos, los primeros no reflejan exactamente la variabilidad de los segundos. Estas ideas, han ido derivando hasta el punto de la necesidad de establecer dentro de cada ecoregión (Gerritsen et al., 2000; Hawkins et al., 2000), ecotipos que incluyan toda la variabilidad, basado en factores locales. Esta es la idea que también utiliza la Directiva Marco del Agua para clasificar los sistemas fluviales (D.O.C.E., 2000).

Cabe destacar que, así como la ecoregión usada para la clasificación comprende un área grande y por tanto posee unos límites (Van Sickle \& Hughes, 2000; Feminella, 2000), el uso de ecotipos no posee estas características ya que están basados en las propiedades físicas y geomorfológicas de un tramo de ríos concreto, propiedades que pueden cambiar aguas abajo. Ello supone que el uso de estos factores más locales, implique una mejor clasificación de los ríos y por tanto de las comunidades, que no las basadas en ecoregiones (Gerritsen et al., 2000; Hawkins \& Norris, 2000; Sandin \& Johnson, 2000).

El uso de la tipología de los ríos, es un paso previo a la determinación del estado ecológico (Barbour et al., 1996; Hawkins et al., 2000; Van Sickle \& Hughes, 2000), ya que es de suponer $a$ priori que las características naturales (en ausencia de contaminación) pueden variar entre distintos tipos de ríos (Hawkins \& Norris, 2000; Oswood et al., 2000), debido a su diferentes parámetros físicos y geomorfológicos (altitud, posición que ocupan dentro de la cuenca, geología,...). Ello por lo tanto, puede tener implicaciones en los índices biológicos, cuyos rangos deberían ser distintos en función del ecotipo, puesto que las comunidades tipo son distintas. 
Un hecho importante a tener en cuenta, es que al tipificar los ríos en un número determinado de grupos, se requiere (y así lo especifica la DMA) un número mínimo de estaciones de referencia por tipo (Chovarec et al., 2000, Hawkins et al., 2000; Moog \& Wimmer, 1990), para poder establecer los rangos de calidad para cada tipo, o bien el máximo potencial ecológico, puesto que para algunos ecotipos, obtener estaciones de referencia estrictas (sin alteraciones antrópicas) puede ser difícil o imposible. El número de ecotipos que se deben establecer, debería pues tener un sentido ecológico, pero a la vez contener un número mínimo de estaciones de referencia. Es por ello que en nuestro estudio se ha descartado el uso del Sistema A para la tipificación, puesto que 16 grupos son demasiados para cumplir ambas condiciones. En cambio los 6 grupos obtenidos por el Sistema B, permiten diferenciar suficientemente los tipos de ríos y establecer programas de estudio y seguimiento viables desde todos los puntos de vista. A pesar de ello, la DMA propone que en caso de que se utilice el Sistema B, para éste se debe lograr, por lo menos, el mismo grado de discriminación que se lograría con el Sistema A, es decir 16 grupos. Ello parece excesivo dado que se deben cumplir también las dos condiciones comentadas. En un estudio previo sobre la tipología de los ríos peninsulares según el Sistema A de la DMA, Marchamalo \& García de Jalón (2000) obtuvieron 35 tipos distintos. A pesar de que hay que destacar que para este estudio se utilizaron 4 tipos de geología en lugar de los 3 que propone la DMA, el elevado número de grupos resultantes indicaría que el Sistema A supondría una complicación excesiva de la gestión sin ninguna base ecológica. Es importante establecer un número óptimo de ecotipos para con ello conseguir una buena clasificación, cuyo objetivo debe estar encaminado a la obtención del estado ecológico de manera fiable (Reynoldson et al., 1997). Así, una correcta clasificación permitirá reducir la probabilidad de cometer un error tipo I (detectar perturbación cuando ésta no existe) y tipo II (no detectar perturbación cuando ésta existe) (Hawkins et al., 2000).
Las variables usadas en este estudio para la tipificación con el Sistema B, son la mayoría de las propuestas en la DMA, y aquellas que se podían calcular con facilidad con los datos que se disponían. Es importante, que los factores que se usen cumplan dos condiciones: que no estén afectados por la actividad humana, y que sean capaces de diferenciar distintos grupos de localidades (Munné \& Prat, 1999, 2000; Gerritsen et al., 2000). La elevada variabilidad geomorfológica y física de los ríos muestreados supone que en algunos casos unas variables sean más importantes para discriminar unos grupos que otras (Pan et al., 2000), tal y como se observa en la figura 2.

Un factor importante a tener en cuenta al tratar los datos mediante esta metodología es el error de mala clasificación, que en nuestro caso podría corresponder a aquellas estaciones englobadas en un ecotipo pero con características de otros. Este error en la clasificación podría ser debido, principalmente a dos factores: los análisis estadísticos y la base de datos original. El método K-means crea grupos de estaciones maximizando las distancias entre ellos, lo que a la práctica puede inducir errores en la clasificación. Por otro lado, la calidad de la base de datos con la que se ha realizado el análisis también puede influir. En nuestro caso, se han utilizado un número de variables incompleto (si comparamos por ejemplo con el trabajo en el Ebro de Munné \& Prat, 1999; 2000), y en algunos casos los valores de cada variable son de tipo cualitativo, lo que indica una menor precisión. Además, ciertas variables que podrían ser de interés para indicar la temporalidad de las estaciones, por ejemplo, no fue posible incluirlas debido a que no están disponibles. Es por ello que, siguiendo la experiencia de los componentes del grupo, se establecieron 3 ecotipos nuevos con propiedades hidrológicas, geomorfológicas y biológicas características: ríos temporales, ríos que nacen en un karst y ríos de la llanura aluvial. Se trata pues, de un ensayo de tipología, que se pretende mejorar en la segunda fase del Proyecto, ampliando la base de datos original incluyendo nuevas variables y precisando las existentes. 
El Sistema B con las modificaciones realizadas presenta una coherencia ecológica con la biología de las comunidades características de cada ecotipo al usar el análisis IndVal. Así por ejemplo, las comunidades de cabecera, están formadas por plecópteros y tricópteros, mientras que en las ramblas, el hábitat lenítico propio de estas zonas permite una gran proliferación de odonatos, heterópteros y coleópteros (Vidal-Abarca, 1990; Moreno et al., 1996; 1997; Vidal-Abarca et al., 1996). En los tramos bajos de ríos medianos o en los de ríos grandes las comunidades tipo son menos diversas que en las cabeceras, debido quizás, a sus características geomorfológicas y también a las actividades humanas que suponen una mayor abundancia de aquellos taxones más generalistas, como los quironómidos y baétidos. A pesar de ello, encontramos organismos interesantes de destacar por su poca ocurrencia como los potamántidos, prosopistómidos, atíidos o nerítidos; juntamente con dugésidos, caénidos, hydropsíchidos,... o los heptagénidos que debido a su sensibilidad a la contaminación solamente se encontrarán en aquellas estaciones de buena calidad. Así pues a pesar de las limitaciones de este ensayo tipológico, los ecotipos obtenidos reflejan de forma bastante clara la diversidad de ríos dentro de la zona mediterránea.

\section{AGRADECIMIENTOS}

Este trabajo se ha realizado mediante la financiación de los proyectos HID98-0323-C05 y REN2001-3438-C07 del Ministerio de Ciencia y Tecnología y PLP/10/FS/97 de la Fundación Séneca de la CARM. Nuestro especial agradecimiento al Area de Coordinación y Aplicaciones Tecnológicas de la D.G.O.H. del Ministerio de Medio Ambiente, al Àrea de Medi Ambient de la Diputació de Barcelona, a la Delegación de Granada de la Consejería de Medio Ambiente de la Junta de Andalucía y a la Agencia Catalana del Aigua por su apoyo.

\section{BIBLIOGRAFÍA}

ABELL, R. A., D. M. OLSON, E. DINERSTEIN, P.T. HURTLEY, J. T. DIGGS, W. EICHBAUM, S. WALTERS, W. WETTENGEL, T. ALLNUTT, C. J. LOUCKS \& P. HEDAO. 2000. Freshwater ecoregions of North America. A conservation assessment. Washington, DC: Island Press.

BAILEY, R. G. 1996. Ecosystem geography. New York: Springer-Verlag.

BAKER, W. L. 1989. Classification of the riparian vegetation of the montane and subalpine zones in western Colorado. Great Basin Naturalist, 9: 214228.

BARBOUR, M. T., J. GERRITSEN, G. E. GRIFFITH, R. FRYDENBORG, E. McCARRON, J. S. WHITE \& M. L. BASTIAN. 1996. A framework for biological criteria for Florida streams using benthic macroinvertebrates. J. N. Am. Benthol. Soc., 15: 185-211.

BONADA, N., N. PRAT, A. MUNNÉ, M. RIERADEVALL, J. ALBA-TERCEDOR, M. ÁLVAREZ, J. AVILÉS, J. CASAS, P. JÁIMEZCUÉlLAR, A. MELLADO, G. MOYÁ, I. PARDO, S. ROBLES, G. RAMÓN, M. L. SUÁREZ, M. TORO, M. R. VIDAL-ABARCA, S. VIVAS \& C. ZAMORA-MUÑOZ. 2002. Criterios para la selección de condiciones de referencia en los ríos mediterráneos. Resultados del proyecto GUADALMED. Limnetica, 21: 99-114.

BOUVET, Y. 1974. Ecologie et reproduction chez les Trichoptères cavernicoles du groupe de Stenophylax (Limnephilidae, Stenophylacini). Proc. of the First Int. Symp. on Trichoptera: 105109.

CHOVAREC, A., P. JÄGER, M. JUNGWIRTH, V. KOLLER-KREIMEL, O. MOOG, S. MUHAR \& ST. SCHMUTZ. 2000. The Austrian way of assessing the ecological integrity of running waters: a contribution to the EU Water Framework Directive. Hydrobiologia, 422/423: 445-452.

D.O.C.E. 2000. Directiva 2000/60/CE del Parlamento Europeo y del Consejo de 23 de octubre de 2000 por la que se establece un marco comunitario de actuación en el ámbito de la politica de aguas. D.O.C.E. L 327 de 22.12.00. $69 \mathrm{pp}$.

DUFRÊNE, M. \& P. LEGENDRE. 1997. Species assemblages and indicator species: the need for a flexible asymmetrical approach. Ecological Monographs, 67: 345-366. 
FEMINELLA, J.W. 2000. Correspondence between stream macroinvertebrate assemblages and 4 ecoregions of the southeastern USA. J. N. Am. Benthol. Soc., 19: 442-461.

FRISSELL, C. A., W.J. LISS, C. E. WARREN \& M. D. HURLEY. 1986. A hierachical framework for stream classification: viewing streams in a watershed context. Environmental Management, 10: 199-214.

GERRITSEN, J., M. T. BARBOUR \& K. KING. 2000. Apples, oranges and ecoregions: on determining pattern in aquatic assemblages. J. N. Am. Benthol. Soc., 19: 497-500.

HAWKINS, C. P. \& R. H. NORRIS. 2000. Performance of different landscape classifications for aquatic bioassessments: introduction to the series. J. N. Am. Benthol. Soc., 19: 367-369.

HAWKINS, C. P. \& M. R. VINSON. 2000. Weak correspondence between landscape classifications and stream invertebrate assemblages: implications for bioassessment. J. N. Am. Benthol. Soc., 19: 501-517.

HAWKINS, C. P., R. H. NORRIS, J. GERRITSEN, R. M. HUGHES, S. K. JACKSON, R. K. JOHNSON \& R. J. STEVENSON. 2000. Evaluation of the use of landscape classifications for the prediction of freshwater biota: synthesis and recommendations. J. N. Am. Benthol. Soc., 19: 541-556.

HOCUTT, C. H. \& E. O. WILEY. 1986. The zoogeography of North American freshwater fishes. New York: John Wiley and Sons.

HURTADO, I., R. GÓMEZ, M. L. SUÁREZ \& M. R. VIDAL-ABARCA. 2001. Typology and environmental characterization of ephemeral streams ("ramblas") of southeastern Spain. Libro de resúmenes del Symposium for European Freshwater Sciences: SEFS-2, Toulouse, Francia: 74.

HYNES, H. B. N. 1975. Edgardo Baldi memorial lecture: the stream and its valley. Verh. Internat. Verein. Theor. Ange. Limnol., 19: 1-15.

ILLIES, J. 1961. Versuch einer allgeimein biozonotischen Gleiderung der Fliessgewasser. Verh. Internat. Verein. Theor. Ange. Limnol., 13: 834844.

ILLIES, J. \& L. BOTOSANEANU. 1963. Problèmes et methods de la classification et de la zonation écologique des eaux courantes considerées surtout du point de vue faunistique. Mitt. Internat. Verein. Theor. Ange. Limnol., 12: 1-57.

ILLIES, J. 1978. Limnofauna europaea. Amsterdam: Swets \& Zeitlinger B. V.
MARCHAMALO, M. \& D. GARCÍA DE JALÓN. 2000. Clasificación ecotipológica de los ríos españoles peninsulares según la Directiva Marco de Política de Agua Europea. Libro de resúmenes del $X$ Congreso de la Asociación Española de Limnología y II Congreso Ibérico de Limnología, Valencia, España: 244.

MARGALEF, R. 1983. Limología. Barcelona: Ediciones Omega.

MCCORMICK, F. H., D. V. PECK \& D. P. LARSEN. 2000. Comparison of geographic classification schemes for Mid-Atlantic stream fish assemblages. J. N. Am. Benthol. Soc., 19: 385-404.

MOOG, O. \& R. WIMMER. 1990. Grundlagen zur typologische Charakteristik österreichischer Fließgewässer. Wasser Abwasser, 34: 55-211.

MORENO, J. L., M.L. SUÁREZ \& M. R. VIDALABARCA. 1996. Valor ecológico de las ramblas como ecosistemas acuáticos singulares. RSEHN Tomo extraordinario. 125 Aniversario: 411-415.

MORENO, J. L., M. L. SUÁREZ, M. R. VIDALABARCA \& J. VELASCO. 1997. Aquatic Coleoptera and Heteroptera assemblages in waterbodies from ephemeral coastal streams ("ramblas") of south-eastern Spain. Arch. für Hydrobiol. 141: 93-107.

MOSS, D., M. T. FURSE, J. F. WRIGTH \& P. D. ARMITAGE. 1987. The prediction of the macroinvertebrate fauna of unpolluted running-water sites in great Britain using environmental data. Freshwat. Biol., 17: 41-52.

MUNNÉ, A. \& N. PRAT. 1999. Regionalización de la cuenca del Ebro para el establecimiento de los objetivos del estado ecológico de sus ríos. Confederación Hidrográfica del Ebro (Oficina de Planificación Hidrológica). Zaragoza. 186 pp.

MUNNÉ, A. \& N. PRAT. 2000. Delimitación de regiones ecológicas para el establecimiento de tipos de referencia y umbrales de calidad biológica. Actas del Segundo Congreso Ibérico sobre Gestión y Planificación del Agua, Oporto, Portugal.

MUNNÉ, A., C. SOLÀ \& N. PRAT. 1998. QBR: Un índice rápido para la evaluación de la calidad de los ecosistemas de ribera. Tecnología del Agua, 175: 20-37.

MUNNÉ, A., N. PRAT, C. SOLÀ, N. BONADA \& M. RIERADEVALL. 2003. A simple field method for assessing the ecological quality of riparian habitat in rivers and streams. QBR index. Aquatic Conserv.: Mar. Freshw. Ecosyst., 13: 147-164. 
NAIMAN, R. J., D.G. LONZARICH, T. J. BEECHE \& S.C. RALPH. 1992. General principles of classification and the assessment of conservation potential in rivers. In: River Conservation and Management. P. J. Boon \& G. E. Petts (eds.): 93123. John Wiley \& Sons Ltd, England.

NAIMAN, R. J., S. R. ELLIOT, J. M. HELFIELD \& T. C. O'KEEFE. 2000. Biophysical interactions and the structure and dynamics of riverine ecosystems: the importance of biotic feedbacks: Hydrobiologia, 410: 79-86

NORRIS, R. H. 1995. Biological monitoring: the dilemma of data analysis. J. N. Am. Benthol. Soc., 14: 440-450.

OMERNIK, J. M. 1987. Ecoregions of the conterminous United States. Annals of the Association of American Geographers, 77: 118-125.

OMERNIK, J. M. 1995. Ecoregions: a framework for managing ecosystems. George Wrigth Forum 12: $35-50$.

OSWOOD, M. W., J.B. REYNOLDS, J. G. IRONS III \& A. M. MILNER. 2000. Distributions of freshwater fishes in ecoregions and hydroregions of Alaska. J. N. Am. Benthol. Soc., 19: 405-418.

PAN, Y., R. J. STEVENSON, B. H. HILL \& A.T. HERLIHY. 2000. Ecoregions and benthic diatom assemblages in Mid-Atlantic Highlands streams, USA. J. N. Am. Benthol. Soc., 19: 518-540.

RABENI, C. F. \& K. E. DOISY. 2000. Correspondence of stream benthic invertebrate assemblages to regional classification schemes in Missouri. J. N. Am. Benthol. Soc., 19: 419-428.

RESH, V. H., R. H. NORRIS \& M. T. BARBOUR. 1995. Design and implementation of rapid assessment approaches for water resource monitoring using benthic macroinvertebrates. Aust. J. Ecol., 20: 108-121.

REYNOLDSON, T. B., R. C. BAILEY, K. E. DAY \& R. H. NORRIS. 1995. Biological guidelines for freshwater sediment based on Benthic Assessment of SedimenT (the BEAST) using a multivariate approach for predicting biological state. Austral. J. Ecol., 20: 198-219.

REYNOLDSON, T. B., R. H. NORRIS, V. H. RESH, K. E. DAY \& D. M. ROSENBERG. 1997. The reference condition: a comparision of multimetric and multivariate approaches to assess water-quality impairment using benthic macroinvertebrates. J. N. Am. Benthol. Soc., 16: 833-852.

SANDIN, L. \& R. K. JOHNSON. 2000. Ecoregions and benthic macroinvertebrate assemblages of
Swedish streams. J. N. Am. Benthol. Soc., 19: 462-474.

SIMPSON, J. C., R. H. NORRIS, L. BARMUTA \& P. BLACKMAN. 1997. Australian River Assessment System. National River Health Program predictive model manual. Belconnen, ACT, Australia: Cooperative Reasearch Centre for Freshwater Ecology, University of Canberra (http://ausrivas.camberra.edu.au/ausrivas/manual.

SUÁREZ, M. L., M. R. VIDAL-ABARCA, M. M. SÁNCHEZ-MONTOYA, J. ALBA-TERCEDOR, M. ÁlVAREZ, J. AVILÉS, N. BONADA, J. CASAS, P. JÁIMEZ-CUÉLLAR, A. MUNNÉ, I. PARDO, N. PRAT, M. RIERADEVALL, M. J. SALINAS, M. TORO \& S. VIVAS. 2002. Las riberas de los ríos mediterráneos y su calidad: El uso del índice QBR. Limnetica, 21: 135-148.

TER BRAAK, J.F. 1988. "CANOCO - a FORTRAN program for canonical community ordenation by (partial) (detrended) (canonical) correspondence analysis, principal components analysis and redundancy analysis (version 2.1).

TORO, M., S. ROBLES, J. AVILÉS, C. NUÑO, S. VIVAS, N. BONADA, N. PRAT, J. ALBATERCEDOR, J. CASAS, C. GUERRERO, P. JÁIMEZ-CUÉLLAR, J. L. MORENO, G. MOYÁ, G. RAMON, M. L. SUÁREZ, M. R. VIDALABARCA, M. ÁlVAREZ \& I. PARDO. 2002. Calidad de las aguas de los ríos mediterráneos del proyecto GUADALMED. Limnetica, 21: 63-75.

VAN SICKLE, J. \& R.M. HUGHES. 2000. Classification strengths of ecoregions, catchments, and geographic clusters for aquatic vertebrates in Oregon. J. N. Am. Benthol. Soc., 19: 370-384.

VIDAL-ABARCA, M. R. 1990. Los ríos de las cuencas áridas y semiáridas: Una perspectiva ecológica comparativa y de síntesis. Scientia gerundensis, 16: 219-228.

VIDAL-ABARCA, M. R., C. MONTES, M. L. SUÁREZ \& L. RAMIÍREZ-DÍAZ. 1990. Sectorización ecológica de cuencas fluviales: Aplicación a la Cuenca del Río Segura (SE. de España). Anales de Geografía de la Universidad Complutense, 10: 149-182.

VIDAL-ABARCA, M. R., M. L. SUÁREZ \& L. RAMÍREZ-DÍAZ. 1996. Tipo: Ramblas/Wadis. En: Management of mediterranean wetlands. III. Case studies 2. Proyecto MEDWET. C. Morillo \& J.L. Gónzalez (eds.): 17-38. Unión Europea. Ministerio de Medio Ambiente. Dirección General de Conservación de la Naturaleza. 
WARREN, C. E. 1979. Toward classification and rationale for watershed management and stream protection. Report No. EPA-600/3-79-059. Corvallis, Oregon: United States Environmental Protection Agency.

WARRY, N. D. \& M. HANAU. 1993. The use of terrestial ecoregions as a regional-scale screen for selecting representative references sites for water quality monitoring. Environmental Management, 17: $267-276$.
WRIGTH, J. F., D. MOSS, P. D. ARMITAGE \& M. T. FURSE. 1984. A preliminary classification of running water sites in Great Britain based on macroinvertebrate species and the prediction of community type using environmental data. Freshwat. Biol., 14: 221-256.

WRIGTH, J. F., M. T. FURSE \& P. D. ARMITAGE. 1993. RIVPACS - a technique for evaluating the biological quality of rivers in the U.K. European Water Pollution Control, 3: 15-25. 
Anexo 1. Clasificación de las estaciones del proyecto GUADALMED según el sistema A de la DMA. Se diferencian las estaciones de referencia según el criterio de los expertos. Classification of the GUADALMED project sampling sites according to WFD System A. Reference localities were selected on the basis of expert judgment are segregated from the non-reference ones.

\begin{tabular}{|c|c|c|c|c|c|}
\hline Sistema A & Referencia & No Referencia & Sistema A & Referencia & No Referencia \\
\hline \multirow[t]{3}{*}{ Alto, Grande, Calcáreo } & & JU9 & \multirow[t]{11}{*}{ Altura media, Pequeño, Calcáreo } & \multirow{11}{*}{$\begin{array}{l}\text { AG2-R } \\
\text { B24-R } \\
\text { GU3-R } \\
\text { L44-R } \\
\text { L45-R } \\
\text { SE8-R }\end{array}$} & B12 \\
\hline & & TU3 & & & $\mathrm{B} 22$ \\
\hline & & TU7 & & & $\mathrm{B} 25$ \\
\hline \multirow{8}{*}{ Alto, Mediano, Calcáreo } & JU1-R & JU17 & & & B28 \\
\hline & JU2-R & MI1 & & & B30 \\
\hline & JU8-R & MI10 & & & GU9 \\
\hline & SE1-R & MI12 & & & P010 \\
\hline & SE2-R & MI3 & & & P06 \\
\hline & TU2-R & MI7 & & & SE11 \\
\hline & TU6-R & & & & S02 \\
\hline & IUO-K & & & & S07 \\
\hline \multicolumn{2}{|l|}{ Alto, Mediano, Silíceo } & AL3 & \multirow{10}{*}{ Altura media, Pequeño, Silíceo } & \multirow{10}{*}{$\begin{array}{l}\text { AD3-R } \\
\text { AD4-R } \\
\text { AG7-R } \\
\text { B32-R } \\
\text { B35-R } \\
\text { B7a-R } \\
\text { B7-R }\end{array}$} & AL10 \\
\hline Alto, Pequeño, Calcáreo & GU2-R & SE4 & & & AL 11 \\
\hline 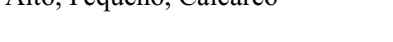 & JU6-R & TU5 & & & AL 12 \\
\hline & JU7-R & & & & AL14 \\
\hline & L56-R & & & & AL15 \\
\hline & SE3-R & & & & AL4 \\
\hline & TU1-R & & & & AL5 \\
\hline & TU4-R & & & & $\mathrm{B} 17 \mathrm{a}$ \\
\hline \multirow[t]{7}{*}{ Alto, Pequeño, Silíceo } & \multirow{7}{*}{$\begin{array}{l}\text { AD5-R } \\
\text { AL6-R } \\
\text { AL7-R } \\
\text { GU14-R } \\
\text { GU15-R } \\
\text { GU1-R } \\
\text { GU5-R } \\
\text { GU6-R }\end{array}$} & \multirow{7}{*}{$\begin{array}{l}\text { AL1 } \\
\text { AL2 } \\
\text { AL8 } \\
\text { GU11 } \\
\text { GU12 }\end{array}$} & & & GU16 \\
\hline & & & & & GU8 \\
\hline & & & Tierras bajas Grande Calcáreo & TU11-R & JU16 \\
\hline & & & 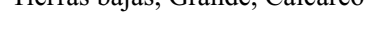 & 1011-10 & L101 \\
\hline & & & & & L102 \\
\hline & & & & & L90 \\
\hline & & & & & L94 \\
\hline \multirow[t]{9}{*}{ Altura media, Grande, Calcáreo } & \multirow{9}{*}{$\begin{array}{l}\text { JU11-R } \\
\text { JU5-R } \\
\text { MI4-R } \\
\text { TU10-R } \\
\text { TU9-R }\end{array}$} & \multirow{9}{*}{$\begin{array}{l}\text { JU10 } \\
\text { JU19 } \\
\text { JU3 } \\
\text { JU4 } \\
\text { L38 } \\
\text { MI11 } \\
\text { MI5 } \\
\text { SE6 } \\
\text { TU13 } \\
\text { TU8 }\end{array}$} & & & MI6 \\
\hline & & & & & \\
\hline & & & Tierras bajas, Mediano, Calcáreo & GU10-R & AG6 \\
\hline & & & & & AL17 \\
\hline & & & & & AL18 \\
\hline & & & Tierras bajas, Mediano, Silíceo & & AD1 \\
\hline & & & & & AG8 \\
\hline & & & & & B16 \\
\hline & & & & & B4 \\
\hline \multirow[t]{11}{*}{ Altura media, Mediano, Calcáreo } & \multirow{11}{*}{$\begin{array}{l}\text { AG1-R } \\
\text { L60a-R } \\
\text { L61-R } \\
\text { MI8-R } \\
\text { MI9-R } \\
\text { SE 10-R } \\
\text { SE 16-R } \\
\text { SE17-R } \\
\text { SE 18-R } \\
\text { SE5-R } \\
\text { SE9-R } \\
\text { TU12-R }\end{array}$} & \multirow{11}{*}{$\begin{array}{l}\text { AD2 } \\
\text { GU4 } \\
\text { JU15 } \\
\text { L42 } \\
\text { L54 } \\
\text { L60c } \\
\text { L64a } \\
\text { L68 } \\
\text { SE7 }\end{array}$} & \multirow{3}{*}{\multicolumn{2}{|c|}{ Tierras bajas, Muy grande, Calcáreo }} & JU13 \\
\hline & & & & & JU14 \\
\hline & & & & & JU18 \\
\hline & & & \multirow{14}{*}{ Tierras bajas, Pequeño, Calcáreo } & \multirow{14}{*}{$\begin{array}{l}\text { P01-R } \\
\text { P07-R } \\
\text { P08-R } \\
\text { P09-R } \\
\text { SE12-R } \\
\text { SE14-R }\end{array}$} & \\
\hline & & & & & AG3 \\
\hline & & & & & AG4 \\
\hline & & & & & AG5 \\
\hline & & & & & P0 11 \\
\hline & & & & & $\mathrm{P} 02$ \\
\hline & & & & & P03 \\
\hline & & & & & $\mathrm{P} 05$ \\
\hline \multirow{5}{*}{ Altura media, Mediano, Silíceo } & & & & & $\begin{array}{l}\text { SE13 } \\
\text { SE15 }\end{array}$ \\
\hline & GU7-R & AL13 & & & SE15 \\
\hline & & AL16 & & & S01 \\
\hline & & AL9 & & & S04 \\
\hline & & $\mathrm{B} 10$ & & & S05 \\
\hline Altura media, Muy grande, Calcár & & JU12 & & & $\begin{array}{l}\text { S06 } \\
\text { S03 }\end{array}$ \\
\hline
\end{tabular}


Anexo 2. Clasificación de las estaciones del proyecto GUADALMED según el Sistema B obtenidas con el K-means. Classification of the sampling sites in project GUADALMED according to WFD System B, obtained using a K-means analysis.

\begin{tabular}{|c|c|c|c|c|c|}
\hline \multicolumn{2}{|c|}{ Cabeceras } & \multicolumn{2}{|c|}{ Tramos medios Bajos } & \multirow{2}{*}{$\begin{array}{l}\text { Ríos grandes } \\
\mathrm{Cal} / \mathrm{GB}\end{array}$} & \multirow{2}{*}{$\begin{array}{l}\text { Ramblas } \\
\text { Sedim }\end{array}$} \\
\hline $\mathrm{Sil} / \mathrm{Cab}$ & $\mathrm{Calc} / \mathrm{Cab}$ & $\mathrm{Sil} / \mathrm{MB}$ & Calc/MB & & \\
\hline AD5-R & B24-R & AD1 & $\mathrm{AD} 2$ & JU10 & AG1-R \\
\hline AL1 & GU2-R & AD3-R & B12 & JU11-R & AG2-R \\
\hline AL10 & JU17 & AD4-R & B22 & JU12 & AG3 \\
\hline AL11 & JU1-R & AG7-R & B25 & JU13 & AG4 \\
\hline AL5 & JU6-R & AL12 & B28 & JU14 & AG5 \\
\hline AL6-R & JU7-R & AL13 & B30 & JU18 & AG6 \\
\hline AL7-R & JU8-R & AL14 & GU3-R & JU3 & AG8 \\
\hline AL8 & L44-R & AL15 & GU4 & JU4 & SE11 \\
\hline B32-R & L45-R & AL16 & JU15 & JU9 & SE12-R \\
\hline B35-R & L56-R & AL17 & JU16 & TU11-R & SE13 \\
\hline B7a-R & MI1 & AL18 & JU19 & TU13 & SE14-R \\
\hline B7-R & MI10 & AL2 & JU2-R & TU8 & SE15 \\
\hline GU11 & MI12 & AL3 & JU5-R & & SE17-R \\
\hline GU12 & MI3 & AL4 & L101 & & SE7 \\
\hline GU13 & MI7 & AL9 & L102 & & SE8-R \\
\hline GU14-R & MI8-R & B10 & L38 & & SE9-R \\
\hline GU15-R & PO10 & B15 & L42 & & \\
\hline GU1 -R & PO1 -R & B16 & L54 & & \\
\hline GU5-R & $\mathrm{PO} 2$ & $\mathrm{~B} 17 \mathrm{a}$ & L60a-R & & \\
\hline \multirow[t]{23}{*}{ GU6-R } & PO5 & B4 & L60c & & \\
\hline & PO6 & GU10-R & L61-R & & \\
\hline & PO7-R & GU16 & L64a & & \\
\hline & PO8-R & GU7-R & L68 & & \\
\hline & SE1-R & GU8 & L90 & & \\
\hline & SE2-R & GU9 & L94 & & \\
\hline & SE3-R & & MI11 & & \\
\hline & SE4 & & MI4-R & & \\
\hline & SO1 & & MI5 & & \\
\hline & $\mathrm{SO} 2$ & & MI6 & & \\
\hline & $\mathrm{SO} 3$ & & MI9-R & & \\
\hline & SO4 & & PO11 & & \\
\hline & SO6 & & PO3 & & \\
\hline & SO7 & & PO9-R & & \\
\hline & TU12-R & & SE10-R & & \\
\hline & TU14 & & SE16-R & & \\
\hline & TU1-R & & SE18-R & & \\
\hline & TU2-R & & SE5-R & & \\
\hline & TU3 & & SE6 & & \\
\hline & TU4-R & & SO5 & & \\
\hline & TU5 & & TU10-R & & \\
\hline & TU6-R & & TU7 & & \\
\hline & & & TU9-R & & \\
\hline
\end{tabular}


Anexo 3. Tipología provisional de las estaciones del proyecto GUADALMED derivada del análisis estadístico K-means y de las correcciones introducidas por el panel de expertos. Preliminary typology of the GUADALMED project localities derived from a Kmeans analysis and input from the expert panel.

\begin{tabular}{|c|c|c|c|c|c|c|c|c|}
\hline \multicolumn{2}{|c|}{ Cabeceras } & \multicolumn{2}{|c|}{ Tramos medios Bajos } & \multirow{2}{*}{$\begin{array}{l}\text { Ríos grandes } \\
\text { Cal/GB }\end{array}$} & \multirow{2}{*}{$\begin{array}{l}\text { Llanura } \\
\text { Aluvial }\end{array}$} & \multirow[t]{2}{*}{ Temporales } & \multirow[t]{2}{*}{ Karst } & \multirow{2}{*}{$\begin{array}{l}\text { Ramblas } \\
\text { Sedim }\end{array}$} \\
\hline $\mathrm{Sil} / \mathrm{Cab}$ & $\mathrm{Cal} / \mathrm{Cab}$ & $\mathrm{Sil} / \mathrm{MB}$ & $\mathrm{Cal} / \mathrm{MB}$ & & & & & \\
\hline AD5-R & $\mathrm{B} 30$ & AD1 & $\mathrm{AD} 2$ & JU10 & JU14 & $\mathrm{B} 24 \mathrm{R}$ & AL6-R & AG3 \\
\hline AL1 & GU2-R & AD3-R & $\mathrm{B} 12$ & JU11-R & JU18 & B7a-R & AL7-R & AG4 \\
\hline AL10 & JU17 & AD4-R & $\mathrm{B} 22$ & JU12 & & B7-R & AG7-R & AG5 \\
\hline AL11 & JU1-R & AL12 & $\mathrm{B} 25$ & JU13 & & MI1 & AG1-R & AG6 \\
\hline AL5 & JU6-R & AL13 & B28 & JU3 & & PO10 & AG2-R & AG8 \\
\hline AL8 & JU7-R & AL14 & GU3-R & JU4 & & PO5 & PO9-R & SE11 \\
\hline B32-R & JU8-R & AL15 & GU4 & JU9 & & PO6 & PO11 & SE12-R \\
\hline B35-R & L44-R & AL16 & JU15 & TU11-R & & SE4 & L45R & SE13 \\
\hline GU11 & L56-R & AL17 & JU16 & & & SO1 & PO1-R & SE14-R \\
\hline GU12 & MI10 & AL18 & JU19 & & & $\mathrm{SO} 2$ & PO7-R & SE15 \\
\hline GU13 & MI12 & AL2 & JU2-R & & & $\mathrm{SO} 3$ & PO8-R & SE17-R \\
\hline GU14-R & MI7 & AL3 & JU5-R & & & $\mathrm{SO} 4$ & & SE7 \\
\hline GU15-R & MI8-R & AL4 & L101 & & & SO6 & & SE8-R \\
\hline GU1-R & SE1-R & AL9 & L102 & & & SO7 & & SE9-R \\
\hline GU5-R & SE2-R & B10 & L38 & & & TU12-R & & \\
\hline GU6-R & SE3-R & B15 & $\mathrm{L} 42$ & & & & & \\
\hline & TU14 & B16 & L54 & & & & & \\
\hline & TU1-R & $\mathrm{B} 17 \mathrm{a}$ & L60a-R & & & & & \\
\hline & TU4-R & B4 & L60c & & & & & \\
\hline & TU5 & GU10-R & L61-R & & & & & \\
\hline & TU6-R & GU16 & L64a & & & & & \\
\hline & & GU7-R & L68 & & & & & \\
\hline & & GU8 & L90 & & & & & \\
\hline & & GU9 & L94 & & & & & \\
\hline & & & MI11 & & & & & \\
\hline & & & MI3 & & & & & \\
\hline & & & MI4-R & & & & & \\
\hline & & & MI5 & & & & & \\
\hline & & & MI6 & & & & & \\
\hline & & & MI9-R & & & & & \\
\hline & & & PO2 & & & & & \\
\hline & & & PO3 & & & & & \\
\hline & & & SE10-R & & & & & \\
\hline & & & SE16-R & & & & & \\
\hline & & & SE18-R & & & & & \\
\hline & & & SE5-R & & & & & \\
\hline & & & SE6 & & & & & \\
\hline & & & SO5 & & & & & \\
\hline & & & TU10-R & & & & & \\
\hline & & & TU13 & & & & & \\
\hline & & & TU2-R & & & & & \\
\hline & & & TU3 & & & & & \\
\hline & & & TU7 & & & & & \\
\hline & & & TU8 & & & & & \\
\hline & & & TU9-R & & & & & \\
\hline
\end{tabular}

